Article

\title{
Targeting the Cryptococcus neoformans var. grubii Cell Wall Using Lectins: Study of the Carbohydrate-Binding Domain
}

\author{
Pamella de Brito Ximenes ${ }^{1}$, Eduardo Isidoro Carneiro Beltrão ${ }^{2}$, \\ Danielle Patrícia Cerqueira Macêdo ${ }^{3}$, Maria Daniela Silva Buonafina ${ }^{1}$, \\ Reginaldo Gonçalves de Lima-Neto ${ }^{4}$ and Rejane Pereira Neves ${ }^{1, *}$
}

1 Department of Mycology, Universidade Federal de Pernambuco (UFPE), Av. Prof. Nelson Chaves, s/nº-Cidade Universitária, Recife 50670-420, Brazil;

E-Mails: pamella_ximenes@hotmail.com (P.B.X.); danielabuonafina@hotmail.com (M.D.S.B.)

2 Department of Biochemistry, Universidade Federal de Pernambuco (UFPE), Av. Prof. Nelson Chaves, s/nº-Cidade Universitária, Recife 50670-420, Brazil; E-Mail: ebeltrao@hotmail.com Department of Pharmaceutical Sciences, Universidade Federal de Pernambuco (UFPE), Av. Prof. Nelson Chaves, s/nº-Cidade Universitária, Recife 50670-420, Brazil; E-Mail: daniellemacedo28@gmail.com

4 Department of Tropical Medicine, Universidade Federal de Pernambuco (UFPE), Av. Prof. Nelson Chaves, s/nº-Cidade Universitária, Recife 50670-420, Brazil; E-Mail: goncalves_reginaldo@hotmail.com

* Author to whom correspondence should be addressed; E-Mail: rejadel@yahoo.com.br; Tel.: +5581-2126-8570; Fax: +5581-2126-8481.

Academic Editor: Tzi Bun NG

Received: 15 November 2014 / Accepted: 16 January 2015 / Published: 25 February 2015

\begin{abstract}
Cryptococcus neoformans var. grubii is considered to be the major cause of cryptococcosis in immunosuppressed patients. Understanding cell wall glycoproteins using lectins is of medical interest and can contribute to specific therapy. The aim of this study was to evaluate the carbohydrates on the cell wall of Cryptococcus neoformans var. grubii clinical isolates, using a fluorescein isothiocyanate-lectin binding protocol. Thirty yeast strains stocked in the culture collection were cultivated for 2 days at $30{ }^{\circ} \mathrm{C}$ with shaking. Cells were obtained by centrifugation, washed in phosphate-buffered saline, and a suspension of $10^{7}$ cells $/ \mathrm{mL}$ was obtained. To determine the binding profile of lectins, concanavalin A (Con A), wheat germ agglutinin (WGA), Ulex europaeus agglutinin I (UEA-I), and peanut agglutinin (PNA) conjugated to fluorescein were used. All the tested
\end{abstract}


clinical isolates of Cryptococcus neoformans var. grubii were intensely stained by WGA, moderately stained by Con A, and weakly stained by PNA and UEA-I. Thus, Cryptococcus can be detected in clinical specimens such as blood and cerebrospinal fluid using the fluorescent lectin WGA, which may be considered as an option for detection in cases of suspected cryptococcosis with low laboratory sensitivity. Future applications may be developed using this basic tool.

Keywords: lectins; Cryptococcus neoformans var. grubii; cell wall carbohydrates; diagnosis

\section{Introduction}

In developed countries, Cryptococcus neoformans var. grubii is considered to be the major cause of cryptococcosis, a serious human infection that results in death in some cases [1-3]. The mechanisms that are involved in its pathogenesis can best be studied by starting with an understanding of cell wall glycoproteins, which are important characteristics of fungi of medical interest [4]. In this context, different lectins have been used to determine a pattern of cellular glycoconjugates in Cryptococcus species, including C. neoformans var. grubii $[5,6]$.

Lectins are a class of proteins that exhibits huge biological and pharmacological potential; lectins were originally identified in plant, animal, and microbe species [7]. These proteins have at least one non-catalytic domain that binds specifically and reversibly to mono- or oligosaccharides [8]. This carbohydrate recognition capacity allows interactions with cell membrane surface glycoconjugates, consequently interfering with various biological events such as infection, cell differentiation, pathogen-host interaction, metastasis, and cell recognition [9-11].

A number of studies have consistently demonstrated that a knowledge of the surface components of C. neoformans is essential to understand cryptococcosis pathogenesis [5]. It is important to consider that the interaction between the yeast cell wall and host tissue receptors allows the first step in the establishment of disease, adhesion [8]. Thus, the targeting of cell wall glycoconjugates such as $\mathrm{N}$-acetyl-D-glucosamine, L-fucose, D-galactose, and glucose/mannose may elucidate many biological and pathological roles that are currently unclear. The expression of $N$-acetyl-D-glucosamine may be analysed using wheat germ agglutinin (WGA), that of methyl- $\alpha$-D-mannoside using concanavalin A (Con A), the L-fucose using Ulex europaeus agglutinin I (UEA-I), and for detection of D-galactose, and glucose/mannose on the cell wall surface, peanut agglutinin (PNA) is considered the most specific lectin [5].

Conventional detection methods for Cryptococcus species include culture isolation, colony morphology, and nutritional characteristics. This fungus may take 24-72 h or even weeks to grow in primary culture. For this reason, the diagnosis of cryptococcosis at the fungal species level is time consuming and delays treatment seelction. Furthermore, these methods are laborious and may eventually provide inconclusive identifications [9].

In this context, the aim of this study was to evaluate the expression of $N$-acetyl-D-glucosamine, methyl- $\alpha$-D-mannoside, L-fucose, D-galactose, and glucose/mannose on the cell wall surface of 
C. neoformans var. grubii through staining protocols using wheat germ agglutinin (WGA), concanavalin A (Con A), Ulex europaeus agglutinin I (UEA-I), and peanut agglutinin (PNA) lectins.

\section{Results and Discussion}

The lectin-binding assays using WGA, Con A, UEA-I, and PNA showed three carbohydrate-specific fluorescent patterns for the C. neoformans var. grubii strains as presented in Table 1.

Table 1. Cryptococcus neoformans var. grubii strains and fluorescence patterns of lectin staining.

\begin{tabular}{|c|c|c|c|c|}
\hline $\begin{array}{c}\text { Cryptococcus neoformans var. Grubii } \\
\text { *URM }\end{array}$ & WGA lectin & Con A lectin & PNA lectin & UEA-I lectin \\
\hline 5809 & +++ & ++ & + & + \\
\hline 5810 & +++ & ++ & + & + \\
\hline 5811 & +++ & ++ & + & + \\
\hline 5813 & +++ & ++ & + & + \\
\hline 5814 & +++ & ++ & + & + \\
\hline 5815 & +++ & ++ & + & + \\
\hline 5816 & +++ & ++ & + & + \\
\hline 5818 & +++ & ++ & + & + \\
\hline 5819 & +++ & ++ & + & + \\
\hline 5820 & +++ & ++ & + & + \\
\hline 5821 & +++ & ++ & + & + \\
\hline 5822 & +++ & ++ & + & + \\
\hline 5823 & +++ & ++ & + & + \\
\hline 5824 & +++ & ++ & + & + \\
\hline 5825 & +++ & ++ & + & + \\
\hline 6895 & +++ & ++ & + & + \\
\hline 6896 & +++ & ++ & + & + \\
\hline 6897 & +++ & ++ & + & + \\
\hline 6898 & +++ & ++ & + & + \\
\hline 6899 & +++ & ++ & + & + \\
\hline 6900 & +++ & ++ & + & + \\
\hline 6901 & +++ & ++ & + & + \\
\hline 6902 & +++ & ++ & + & + \\
\hline 6903 & +++ & ++ & + & + \\
\hline 6904 & +++ & ++ & + & + \\
\hline 6905 & +++ & ++ & + & + \\
\hline 6906 & +++ & ++ & + & + \\
\hline 6907 & +++ & ++ & + & + \\
\hline 6908 & +++ & ++ & + & + \\
\hline 6909 & +++ & ++ & + & + \\
\hline
\end{tabular}

Notes: Strains were obtained from the Universidade Recife Micologia Culture Collection of Pernambuco (*URM), Brazil; WGA: wheat germ agglutinin; Con A: concanavalin A; PNA: peanut agglutinin; UEA-I: Ulex europaeus agglutinin I.; +++: intense staining pattern; ++: moderate staining pattern; +: weak staining pattern.

The assays were carried out in duplicate, and the duplicates exhibited the same staining patterns. The patterns were intense, moderate, and weak, respectively (Figure 1). 


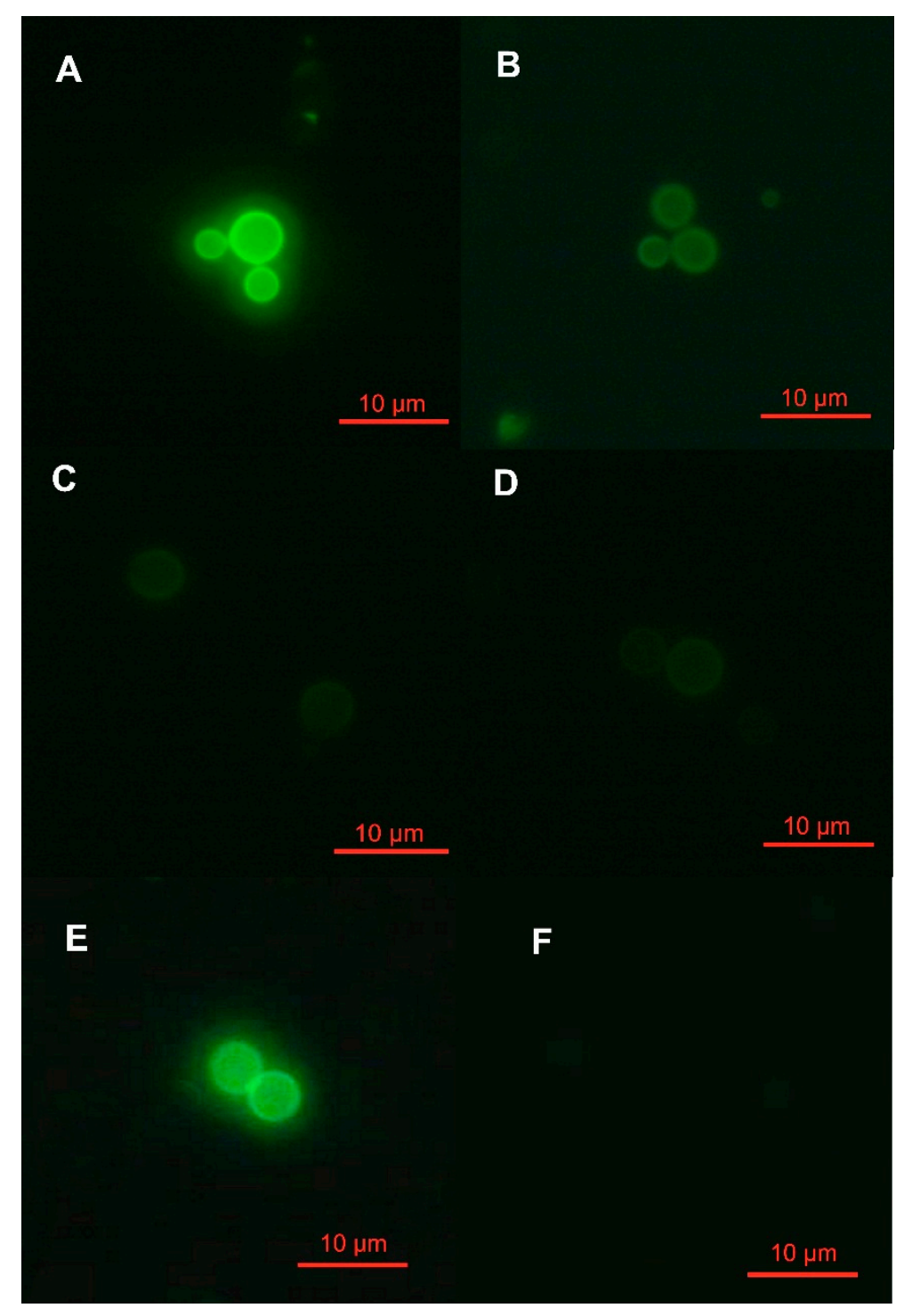

Figure 1. Fluorescence patterns of lectin staining from clinical isolates of Cryptococcus neoformans var. grubii. The staining was intense (A) with wheat germ agglutinin (WGA), moderate (B) with concanavalin A (Con A), and weak (C,D) with peanut agglutinin (PNA) and Ulex europaeus agglutinin I (UEA-I). Positive (E) and negative (F) controls of the fluorescence patterns.

All of the tested strains of $C$. neoformans var. grubii had intense staining with WGA, moderate staining with Con A, and weak staining with PNA and UEA-I when compared with positive and negative controls. These data suggested that $N$-acetyl-D-glucosamine was highly expressed on the cell wall surface of the tested Cryptococcus strains because this carbohydrate is the target molecule of WGA. In contrast, moderate and weak expression of methyl- $\alpha$-D-mannoside and D-galactose/L-fucose were visualized by Con A and PNA/UEA-I staining, respectively.

According to Fonseca et al. [10], the intimate association of cryptococcal cell walls with a distinctive enveloping capsule hinders the isolation and purification of cell wall constituents, thereby compromising our understanding of the cryptococcal cell wall composition, structure, and associated biochemistry. Therefore, analysis of the cell wall composition using fluorescent lectin-staining patterns may facilitate the knowledge and perception of these molecules in situ. 
Previous studies indicated that a typical basidiomycete fungal cell wall (as verified in Cryptococcus species) predominantly comprises $\mathrm{N}$-acetyl glucosamine in the form of chitin microfibrils with gel-like glycoproteins, many of a mannose nature [11]. The Cryptococcus capsule is predominantly comprised of mannose and has been shown to elicit and modulate immune responses in humans $[12,13]$. The most common capsular mannose in Cryptococcus is the polysaccharide glucoronoxylomannan, which forms up to $88 \%$ of the capsular material [13].

Imaging of fluorescein isothiocyanate (FITC)-WGA (Figure 1A), FITC-Con A (Figure 1B), FITC-PNA (Figure 1C), and FITC-UEA-I (Figure 1D) on the cell surface by fluorescence microscopy demonstrated three different staining patterns: intense, moderate, and weak. Based on the results presented in Table 1, the marked fluorescence from the 30 C. neoformans var. grubii strains exposed to FITC-WGA indicates a high concentration of $N$-acetyl-D-glucosamine at the cell surface. In contrast, moderate fluorescence was detected with FITC-Con A in all of the tested strains. According to Foster et al. [13], Con A primarily binds to mannose residues. Additionally, a weak fluorescent pattern was observed in all tested strains when exposed to FITC-PNA and FITC-UEA-I.

Fonseca et al. [10] affirm that the abundance of a particular carbohydrate in the C. neoformans cell wall is related to the strain and growth phase. Some authors suggest that the nature of the FITC-WGA and FITC-Con A exposure appears to be a defining Cryptococcus characteristic [13]. FITC-PNA and FITC-UEA-I exhibited the same results with respect to the fluorescence intensity. Future studies of the C. neoformans var. grubii carbohydrate composition of the cell wall may select between FITC-PNA and FITC-UEA-I because the staining patterns were similar.

Thus, the detection of this species in clinical specimens such as blood and cerebrospinal fluid using the fluorescent lectin WGA may be considered an option for laboratory analyses, especially in cases of suspected meningitis or in immunosuppressed patients with a deficient serologic response. This basic technique may also be used with flow cytometry to obtain a rapid and reliable diagnosis with characterization of the involved species.

\section{Experimental Section}

\subsection{Cryptococcus Strains and Growth Conditions}

The 30 C. neoformans var. grubii strains used in this study were obtained from patients diagnosed with cryptococcosis and have been preserved in the Universidade Recife Micologia Culture Collection (Federal University of Pernambuco, Recife, PE, Brazil) since 2008 as a lyophilized material under mineral oil. Initially, the yeast cells were inoculated into $100-\mathrm{mL}$ Erlenmeyer flasks containing $50 \mathrm{~mL}$ minimal medium composed of $15 \mathrm{mM}$ dextrose, $10 \mathrm{mM} \mathrm{MgSO}_{4}, 29.4 \mathrm{mM} \mathrm{KH}_{2} \mathrm{PO}_{4}, 13 \mathrm{mM}$ glycine, and $3 \mu \mathrm{M}$ thiamine-HCl, $\mathrm{pH}$ 5.5. Fungal cells were cultivated for 2 days at $30^{\circ} \mathrm{C}$ with shaking. The cryptococcal cells were collected by centrifugation, washed in phosphate-buffered saline (PBS), and counted in a Neubauer chamber to obtain a standard suspension of $10^{7}$ cells $/ \mathrm{mL}$ following the method of Rodrigues et al. [9]. 


\subsection{Cryptococcal Cell Wall Lectin Binding}

The binding profile of lectins to the cryptococcal cell wall was verified through the use of Con A, WGA, UEA-I, and PNA conjugated to FITC. First, $1 \mathrm{~mL}$ from the previously obtained standard suspension of the yeast cells in PBS was centrifuged at $470 \mathrm{~g}$ in microfuge tubes for $3 \mathrm{~min}$. Following this step, the pellet was suspended in $0.1 \%$ trypsin for $3 \mathrm{~min}$ at $37{ }^{\circ} \mathrm{C}$ and washed again by centrifugation in PBS. This procedure was repeated twice. After this step, the $25 \mu \mathrm{g} / \mathrm{mL}$ ConA, WGA, UEA, and PNA lectins conjugated to FITC (FITC-lectin) were added separately to the yeast cells obtained after sequential washes for incubation at $4{ }^{\circ} \mathrm{C}$ for $1 \mathrm{~h}$. The preparation was centrifuged, and the pellet was washed with PBS to evaluate the lectin specificity patterns using a fluorescence microscope (AXIO Imager M2m, Zeiss, Calgary, AB, Canada). The binding profiles were expressed according to the fluorescence intensity as follows: intense, +++; moderate, ++; and weak, + . All procedures were repeated twice.

Particularly for the use of WGA lectin, one additional step was developed to prepare samples for binding evaluation. This step involved an enzymatic pre-treatment with neuraminidase to prevent non-specific binding to sialic acids. Specifically, $100 \mu \mathrm{L}$ neuraminidase (Sigma, St. Louis, MO, USA) was added to the yeast suspension after the washes and incubated for $1 \mathrm{~h}$ at $37^{\circ} \mathrm{C}$ before the addition of the lectin according to the previously described steps.

\section{Acknowledgments}

The authors thank the Keiso Asami Immunopathology Laboratory (LIKA, Recife, PE, Brazil) for the use of the electronic microscopy laboratory facilities. We also thank Coordenação de Aperfeiçoamento de Pessoal de Nível Superior (CAPES, Brazil) for financial support.

\section{Author Contributions}

P.B.X. performed the experiments. M.D.S.B. and R.G.L.-N. provided laboratory support. E.I.C.B. and R.P.N. analyzed the data. R.P.N. and D.P.C.M. wrote and edited the manuscript.

\section{Conflicts of Interest}

The authors declare no conflict of interest.

\section{References}

1. Liu, T.B.; Perlin, D.; Xue, C. Molecular mechanisms of cryptococcal meningitis. Virulence 2012, 3, 173-181.

2. Vu, K.; Eigenheer, R.A.; Phinney, B.S.; Gelli, A. Cryptococcus neoformans promotes its transmigration into the central nervous system by inducing molecular and cellular changes in brain endothelial cells. Infect. Immun. 2013, 81, 3139-3147.

3. Santi, L.; Beys-da-Silva, W.O.; Berger, M.; Calzolari, D.; Guimarães, J.A.; Moresco, J.J.; Yates, J.R. Proteomic profile of Cryptococcus neoformans biofilm reveals changes in metabolic processes. J. Proteome Res. 2014, 13, 1545-1559. 
4. Leal, A.F.G.; Lima-Neto, R.G.; Macêdo, D.P.C.; Beltrão, E.I.C.; Neves, R.P. Carbohydrate profile of fungal cell wall surfasse glycoconjugates of Trichophyton tonsurans and other keratinophilic filamentous fungi using lectins. Mycoses 2011, 54, 789-794.

5. Patil, R.T.; Sangwan, J.; Juya, D.; Lathwa, S. Meningitis due to Cryptococcus gattii in an immunocompetent patient. J. Clin. Diagn. Res. 2013, 7, 2274-2275.

6. Seco-Rovira, V.; Beltrán-Frutos, E.; Ferrer, C.; Sánchez-Huertas, M.M.; Madrid, J.F.; Saez, F.J.; Pastor, L.M. Lectin histochemistry as a tool to identify apoptotic cells in the seminiferous epithelium of Syrian hamster (Mesocricetus auratus) subjected to short photoperiod. Reprod. Domest. Anim. 2013, 48, 974-983.

7. Rodríguez-Cerdeira, C.; Arenas, R.; Moreno-Coutiño, G.; Vásquez, E.; Fernández, R.; Chang, P. Micosis sistémicas en pacientes con virus de la inmunodeficiencia humana/SIDA. Actas Dermosifiliogr. 2014, 105, 5-17.

8. Lima-Neto, R.G.; Beltrão, E.I.; Oliveira, P.C.; Neves, R.P. Adherence of Candida albicans and Candida parapsilosis to epithelial cells correlates with fungal cell surface carbohydrates. Mycoses 2011, 54, 23-29.

9. Rodrigues, M.L.; Alvarez, M.; Fonseca, F.L.; Casadevall, A. Binding of the wheat germ lectin to Cryptococcus neoformans suggests an association of chitinlike structures with yeast budding and capsular glucuronoxylomannan. Eukaryot. Cell 2008, 7, 602-609.

10. Fonseca, F.L.; Guimarães, A.J.; Kmetzsch, L.; Dutra, F.F.; Silva, F.D.; Taborda, C.P.; Araujo, G.S.; Frases, S.; Staats, C.C.; Bozza, M.T.; et al. Binding of the wheat germ lectin to Cryptococcus neoformans chitooligomers affects multiple mechanisms required for fungal pathogenesis. Fungal Genet. Biol. 2013, 60, 64-73.

11. Gooday, G.W. Cell walls. In The Growing Fungus; Gow, N.A.R., Gadd, G.M., Eds.; Chapman Hall: London, UK, 1995; pp. 43-62.

12. Pietrella, D.; Cherniak, D.; Strappani, C.; Perito, S.; Mosci, P.; Bistoni, F.; Vecchiarelli, A. Role of mannoprotein in induction and regulation of immunity to Cryptococcus neoformans. Infect. Immun. 2001, 69, 2808-2814.

13. Foster, A.J.; Bird, R.A.; Kelly, S.L.; Nishimura, K.; Poyner, D.; Taylor, S.; Smith, S.N. FITC-lectin avidity of Cryptococcus neoformans cell wall and capsular components. Mycologia 2004, 96, 1-8.

Sample Availability: Samples of the compounds are not available from the authors.

(C) 2015 by the authors; licensee MDPI, Basel, Switzerland. This article is an open access article distributed under the terms and conditions of the Creative Commons Attribution license (http://creativecommons.org/licenses/by/4.0/). 